\title{
SELETIVIDADE DO HERBICIDA METRIBUZIN APLICADO EM PRÉ- EMERGÊNCIA EM CULTIVARES DE AMENDOIM
}

Lorena Regina da Silva Peres ${ }^{1}$; Willians César Carrega ${ }^{2}$; Pablo Nascimento de Oliveira França $a^{3}$; Neriane Hijano ${ }^{4}$; Renan Fabrízzio Lima Viche ${ }^{5}$; Pedro Luis da Costa Aguiar Alves ${ }^{6}$

'Mestra em Aquicultura - Unesp/Caunesp, lorennaperess@hotmail.com; 리outor em Agronomia (Produção Vegetal), FCAV/UNESP, Jaboticabal, SP; ${ }^{3}$ Mestrando em Agronomia (Ciência do Solo), FCAV/UNESP, Jaboticabal, SP; ${ }^{4}$ Doutoranda em Agronomia (Produção Vegetal), FCAV/UNESP, Jaboticabal, SP; ${ }^{5}$ Graduando em Agronomia (Produção Vegetal), FCAV/UNESP, Jaboticabal, SP; ${ }^{6}$ Docente da FCAV/UNESP, Jaboticabal, SP.

RESUMO: Dentre os fatores que interferem no desenvolvimento da cultura do amendoim, a competição com plantas daninhas é considerada de grande importância, sendo o controle químico o principal meio de controle. Pesquisas recentes com herbicidas ainda não registrados para cultura, têm mostrado bastante eficiência no controle e na tolerância pelas cultivares aos produtos. Portanto, objetivou-se avaliar a seletividade do herbicida metribuzin aplicado em pré-emergência, em cultivares de amendoim. O delineamento experimental foi inteiramente casualizado, em esquema fatorial $3 \times 4$, tendo como tratamentos 3 cultivares alto oleicas (IAC OL4, IAC 503 e IAC 505) e 4 doses ( $0 \%, 25 \%$, $50 \%$ e $100 \%$ ) do herbicida metribuzin aplicado em pré-emergência, com quatro repetições. Realizouse avaliações visuais de fitointoxicação aos 15, 21 e 30 dias após a aplicação das doses do metribuzin. Com base nos resultados, constatou-se que as doses de $25 \%, 50 \%$ e $100 \%$ causaram intoxicação interferindo na produção de massa seca da parte aérea e da raiz da cultivar IAC 505. Para a IAC OL4, as doses de $50 \%$ e $100 \%$ causaram fitointoxicação, interferindo na produção de massa seca da raiz. Entre as cultivares, a IAC 503 é a mais tolerante ao herbicida metribuzin, no entanto, nas maiores doses $(50 \%$ e $100 \%)$ ocorreu redução na massa seca da parte aérea e da raiz. Nas condições testadas, o metribuzin apresenta respostas diferenciadas, não sendo seletivo para essas cultivares de amendoim. Entretanto, esse estudo é preliminar e há a necessidade de novas pesquisas, principalmente em campo, avaliando o rendimento, para confirmar as respostas obtidas no presente estudo.

Palavras-chave: Arachis hypogaea L., fitotoxicidade, manejo, plantas daninhas.

\section{INTRODUÇÃO}

O amendoim (Arachis hypogaea L.) é originário da América do Sul e atualmente é encontrado em várias partes do mundo. No Brasil, o seu cultivo está entre as principais oleaginosas (SANTOS et al., 2012), sendo a região sudoeste a principal produtora de amendoim.

Em nível nacional, na última safra (2017/18), o Estado de São Paulo foi responsável por 90\% do total produzido, com cerca de 505,2 mil toneladas (CONAB, 2018). Contudo, a interferência 
16 e 17 de agosto de 2018, centro de convenções da FCAV/UNESP - Câmpus de Jaboticabal, SP

ocasionada pela competição com plantas daninhas é um dos fatores que pode comprometer drasticamente o rendimento dessa cultura. Pesquisas mostraram que a interferência de plantas daninhas com o amendoim podem causar reduções que variam entre $74 \%$ e $92 \%$ (NEPOMUCENO et al., 2007; AGOSTINHO et al., 2006).

Entre as opções de manejo de plantas daninhas, o controle químico é uma das opções mais viáveis, devido a praticidade, eficiência e rapidez na execução. Entretanto, no Brasil, existem poucos herbicidas registrados para a cultura do amendoim, diminuindo as opções para um manejo adequado da cultura. Recentemente, o amendoim foi incluído no MAPA como uma Cultura com Suporte Fitossanitário Insuficiente (MAPA, 2015), possibilitando a abertura do portfólio de outras culturas a serem utilizadas no amendoim (ZANARDO et al., 2018). Assim, o objetivo do trabalho foi avaliar a seletividade do herbicida metribuzin aplicado em pré-emergência em cultivares de amendoim.

\section{MATERIAL E MÉTODOS}

O experimento foi conduzido no Laboratório de Plantas Daninhas (LAPDA), localizado na FCAV/UNESP, Câmpus de Jaboticabal, São Paulo. O delineamento experimental foi inteiramente casualizado (DIC), em esquema fatorial $3 \times 4$, tendo como tratamentos 3 cultivares alto oleicas (IAC OL4, IAC 503 e IAC 505) e 4 doses $(0 \%, 25 \%, 50 \%$ e 100\%) do herbicida metribuzin ( $480 \mathrm{~g} / \mathrm{L})$, com quatro repetições. As doses utilizadas, foram de acordo com a dose recomendada para a cultura da soja, outra espécie da família Fabaceae $\left(1 \mathrm{~L} \mathrm{ha}^{-1}\right)$. Para a realização do experimento, foram utilizados copos de plástico com capacidade volumétrica de $500 \mathrm{ml}$, preenchidos com Latossolo Vermelho escuro de textura média e areia na proporção $(2: 1)$.

As sementes foram previamente tratadas com o fungicida Vitavax ${ }^{\circledR}-T h i r a m ~ 200$ SC (carboxina + thiram) na dosagem de $250 \mathrm{~mL}$ do p.c. $/ 100 \mathrm{~kg}^{-1}$ de sementes para evitar a interferência de patógenos. Logo após a semeadura realizou-se a aplicação em pré-emergência do herbicida, utilizando um pulverizador costal pressurizado por $\mathrm{CO}_{2}$, com volume de calda correspondente a $200 \mathrm{~L} \mathrm{ha}^{-1}$.

As avaliações de fitointoxicação foram realizadas visualmente aos 15, 21 e 30 dias após a aplicação (DAA) do metribuzin em pré-emergência. Para a avaliação, utilizou-se a escala proposta pela EWRC (1964), onde, nota 1, representou a ausência de sintomas e a nota 9, a destruição total das plantas. Após a última avaliação, aos 30 DAA, coletou-se as plantas de cada tratamento, separando-as em parte aérea e raiz e, em seguida, as plantas foram submetidas a secagem em estufa com circulação forçada de ar à uma temperatura entre 65 e $70^{\circ} \mathrm{C}$, até atingir massa constante, para determinação da massa seca da parte aérea e da raiz. Os dados obtidos foram submetidos à análise de variância pelo teste $\mathrm{F}$, e as médias, comparadas pelo teste de Tukey a $5 \%$ de probabilidade. 


\section{RESULTADOS E DISCUSSÃO}

Com base na análise estatística, observou-se que aos 15 DAA da aplicação do metribuzin não foram constatados sintomas de intoxicação para as três cultivares avaliadas no presente estudo. Contudo, aos 21 DAA e 30 DAA, observou-se sintomas de intoxicação, causando interação entre os fatores doses versus cultivares para as notas de sintomas, massa seca da parte aérea e massa seca da raiz (Tabela 1).

Tabela 1. Notas de fitotoxicidade, massa seca da parte aérea (MSPA) e da raiz (MSRA) de plantas de amendoim submetidas a doses do herbicida metribuzin aplicadas em pré-emergência.

\begin{tabular}{llllll}
\hline \hline Cultivares (C) & $15 \mathrm{DAA}$ & 21 DAA & $30 \mathrm{DAA}$ & MSPA & MSRA \\
\hline IAC OL4 & $1,00 \mathrm{~A}$ & $1,56 \mathrm{~B}$ & $3,94 \mathrm{~A}$ & $0,49 \mathrm{~A}$ & $0,14 \mathrm{~A}$ \\
IAC 503 & $1,00 \mathrm{~A}$ & $1,25 \mathrm{C}$ & $1,31 \mathrm{~B}$ & $0,38 \mathrm{~B}$ & $0,11 \mathrm{~B}$ \\
IAC 505 & $1,00 \mathrm{~A}$ & $2,19 \mathrm{~A}$ & $3,50 \mathrm{~A}$ & $0,44 \mathrm{AB}$ & $0,15 \mathrm{~A}$ \\
\hline Doses (D) & & & & & \\
\hline $0 \%$ Dr. & $1,00 \mathrm{~A}$ & $1,08 \mathrm{~B}$ & $1,17 \mathrm{C}$ & $0,64 \mathrm{~A}$ & $0,23 \mathrm{~A}$ \\
$25 \%$ Dr. & $1,00 \mathrm{~A}$ & $1,17 \mathrm{~B}$ & $1,08 \mathrm{C}$ & $0,49 \mathrm{~B}$ & $0,12 \mathrm{~B}$ \\
$50 \%$ Dr. & $1,00 \mathrm{~A}$ & $2,08 \mathrm{~A}$ & $3,33 \mathrm{~B}$ & $0,41 \mathrm{~B}$ & $0,12 \mathrm{~B}$ \\
$100 \%$ Dr. & $1,00 \mathrm{~A}$ & $2,33 \mathrm{~A}$ & $6,08 \mathrm{~A}$ & $0,21 \mathrm{C}$ & $0,07 \mathrm{C}$ \\
\hline F (C) & --- & $29,17^{* *}$ & $49,53^{* *}$ & $3,03 \mathrm{~ns}$ & $7,91^{* *}$ \\
\hline F (D) & --- & $38,67^{* *}$ & $104,09^{* *}$ & $28,14^{* *}$ & $56,12^{* *}$ \\
\hline F (CxD) & --- & $16,50^{* *}$ & $31,79^{* *}$ & $2,44^{*}$ & $3,65^{* *}$ \\
\hline C.V. $(\%)$ & --- & 21,21 & 27,40 & 26,89 & 23,45 \\
\hline \hline
\end{tabular}

Médias seguidas de mesma letra na coluna não diferem entre si ao nível de 5\% de probabilidade pelo Teste de Tukey. Pelo teste $\mathrm{F}$, **significativo a $1 \%$ de probabilidade; *significativo a $5 \%$ de probabilidade. Dr., dose recomendada; C.V. (\%), coeficiente de variação; DAA, dias após aplicação.

Comparando a interação entre as doses para cada cultivar, observou-se que aos 21 DAA, as doses de 50\% e 100\% causaram leve intoxicação para a cultivar IAC OL4 e IAC 503. Para a cultivar IAC 505, verificou-se que a partir da dose de $25 \%$, surgiram leves danos de intoxicação nas plantas, sendo essa injúria mais acentuados quando utilizou-se a dose de 100\%, no entanto, ainda nessa época, os danos foram considerados regulares (com nota 4,00) de acordo com a escala proposta pela EWRC (1964). Comparando as cultivares para cada dose, observou-se diferença apenas para a dose de $100 \%$, com a cultivar IAC 505, seguido da IAC OL4 as que apresentaram maior sensibilidade ao metribuzin (Tabela 2a).

Para a avaliação realizada aos 30 DAA, constatou-se comportamento semelhante aos observados aos 21 DAA, entretanto, ocorreu maior intoxicação das plantas. Entre as cultivares, observou-se que a dose de $50 \%$ promoveu danos médios de intoxicação e a dose de $100 \%$ causou injúria muito forte para a cultivar IAC OL4 e a morte das plantas da cultivar IAC 505 (Tabela 2b), afetando drasticamente a produção de massa seca da parte aérea (Tabela 2c). Para essas duas cultivares, o metribuzin aplicado nas doses de $25 \%, 50 \%$ e $100 \%$ também reduziram a massa seca da 
16 e 17 de agosto de 2018, centro de convenções da FCAV/UNESP - Câmpus de Jaboticabal, SP

raiz (Tabela 2d). Para a cultivar IAC 503 aos 30 DAA, não se observou diferença significativa entre as doses do metribuzin (Tabela $2 b)$, entretanto, nas doses mais altas (50\% e 100\%) verificou-se redução na produção de massa seca da parte aérea e da raiz (Tabela 2c e 2d). Corroborando os resultados evidenciados nesta pesquisa, RAMAKRISHNA et al. (1990) mencionaram que o uso de metribuzin foi tóxico para o amendoim. Segundo HEGDE et al. (1977), o metribuzin causou sérias injúrias em plantas de amendoim, sendo letal para a cultura. Em um experimento com latossolo vermelho-escuro, MARTINS et al. (1980) verificaram que a aplicação de metribuzin em pré-emergência não melhorou os rendimentos do amendoim, quando comparado com o tratamento sem o herbicida. O metribuzin é um herbicida que causa inibição do fotossistema II (PSII), essa inibição ocorre após a absorção do herbicida pela planta e atuam sobre a membrana do cloroplasto, causando a redução dos pigmentos fotossintéticos e promovendo a inibição da fase luminosa, causando a redução do transporte de elétrons (RODRIGUES \& ALMEIDA, 2011). ZANARDO et al. (2018) verificaram que amicarbazone, hexazinone e tebuthiuron, outros herbicidas que inibem o PSII também causaram fortes injúrias nas plantas, levando-as à morte.

Tabela 2. Desdobramento da interação entre os fatores cultivar versus doses para plantas de amendoim submetidas a doses do herbicida metribuzin aplicadas em pré-emergência.

\begin{tabular}{|c|c|c|c|c|}
\hline \multirow{2}{*}{ Doses } & IAC OL4 & IAC 503 & IAC 505 & $\mathrm{~F}$ \\
\hline & \multicolumn{3}{|c|}{21 DAA (a) } & \\
\hline $0 \%$ Dr. & $1,00 \mathrm{Ba}$ & $1,00 \mathrm{Ba}$ & $1,25 \mathrm{Ca}$ & $0,67 \mathrm{~ns}$ \\
\hline $25 \%$ Dr. & $1,00 \mathrm{Ba}$ & $1,00 \mathrm{Ba}$ & $1,50 \mathrm{BCa}$ & $2,67 \mathrm{~ns}$ \\
\hline $50 \%$ Dr. & 2,25 Aa & $2,00 \mathrm{Aa}$ & $2,00 \mathrm{Ba}$ & $0,67 \mathrm{~ns}$ \\
\hline $100 \%$ Dr. & $2,00 \mathrm{Ab}$ & $1,00 \mathrm{Bc}$ & $4,00 \mathrm{Aa}$ & $74,67 * *$ \\
\hline $\mathrm{F}$ & $13,83^{* *}$ & $8,00^{* *}$ & $49,83 * *$ & ----- \\
\hline \multicolumn{5}{|c|}{30 DAA (b) } \\
\hline $0 \%$ Dr. & $1,00 \mathrm{Ca}$ & $1,00 \mathrm{Aa}$ & $1,50 \mathrm{Ba}$ & $0,52 \mathrm{~ns}$ \\
\hline $25 \%$ Dr. & $1,00 \mathrm{Ca}$ & $1,00 \mathrm{Aa}$ & $1,25 \mathrm{Ba}$ & $0,13 \mathrm{~ns}$ \\
\hline $50 \%$ Dr. & $5,50 \mathrm{Ba}$ & $2,25 \mathrm{Ab}$ & $2,25 \mathrm{Bb}$ & $22,04 * *$ \\
\hline $100 \%$ Dr. & $8,25 \mathrm{Aa}$ & $1,00 \mathrm{Ab}$ & $9,00 \mathrm{Aa}$ & $122,22 * *$ \\
\hline $\mathrm{F}$ & $79,92 * *$ & $2,45 n s$ & $85,30 * *$ & ----- \\
\hline \multicolumn{5}{|c|}{ MSPA (c) } \\
\hline $0 \%$ Dr. & $0,62 \mathrm{Aa}$ & $0,56 \mathrm{Aa}$ & $0,74 \mathrm{Aa}$ & $2,34 \mathrm{~ns}$ \\
\hline $25 \%$ Dr. & $0,63 \mathrm{Aa}$ & $0,44 \mathrm{Abab}$ & $0,40 \mathrm{Bb}$ & $4,33 *$ \\
\hline $50 \%$ Dr. & $0,51 \mathrm{Aa}$ & $0,29 \mathrm{Bb}$ & 0,41 Bab & $3,46^{*}$ \\
\hline $100 \%$ Dr. & $0,19 \mathrm{Ba}$ & $0,24 \mathrm{Ba}$ & $0,20 \mathrm{Ba}$ & $0,21 \mathrm{~ns}$ \\
\hline $\mathrm{F}$ & $12,20 * *$ & $6,26 * *$ & $14,56^{* *}$ & ----- \\
\hline \multicolumn{5}{|c|}{ MSRA (d) } \\
\hline $0 \%$ Dr. & $0,27 \mathrm{Aa}$ & $0,16 \mathrm{Ab}$ & $0,25 \mathrm{Aa}$ & $12,83 * *$ \\
\hline $25 \%$ Dr. & $0,13 \mathrm{Ba}$ & $0,11 \mathrm{Aa}$ & $0,14 \mathrm{Ba}$ & $2,89 \mathrm{~ns}$ \\
\hline $50 \%$ Dr. & $0,10 \mathrm{Ba}$ & $0,09 \mathrm{Ba}$ & $0,15 \mathrm{Ba}$ & $2,50 \mathrm{~ns}$ \\
\hline $100 \%$ Dr. & $0,08 \mathrm{Ba}$ & $0,07 \mathrm{Ba}$ & $0,05 \mathrm{Ca}$ & $0,65 \mathrm{~ns}$ \\
\hline $\mathrm{F}$ & $30,24 * *$ & $6,97 * *$ & $26,23 * *$ & ---- \\
\hline
\end{tabular}

Médias seguidas de mesma letra, maiúsculas na coluna e minúsculas na linha não diferem entre si ao nível de $5 \%$ de probabilidade pelo Teste de Tukey. Pelo teste $\mathrm{F}$, **significativo a $1 \%$ de 
16 e 17 de agosto de 2018, centro de convenções da FCAV/UNESP - Câmpus de Jaboticabal, SP

probabilidade; *significativo a $5 \%$ de probabilidade; ns, não significativo. Dr., dose recomendada; C.V. (\%), coeficiente de variação; DAA, dias após aplicação.

\section{CONCLUSÃO}

As doses de $25 \%, 50 \%$ e $100 \%$ causam intoxicação interferindo na produção de massa seca da parte aérea e da raiz da cultivar IAC 505.

Para a cultivar IAC OL4, as doses de 50\% e 100\% promove fitointoxicação, interferindo na produção de massa seca da raiz.

Entre as cultivares, a IAC 503 é a mais tolerante ao herbicida metribuzin, no entanto, nas maiores doses (50\% e 100\%) ocorre redução na massa seca da parte aérea e da raiz.

Nas condições testadas, o metribuzin apresenta respostas diferenciadas, não sendo seletivo para essas cultivares de amendoim. Entretanto, esse estudo é preliminar e há a necessidade de novas pesquisas, principalmente em campo, avaliando o rendimento, para confirmar as respostas obtidas no presente estudo.

\section{REFERÊNCIAS BIBLIOGRÁFICAS}

Agostinho, F. H.; GRAVEnA, R.; AlVES, P. L. C. A.; SAlgAdO, T. P.; MATTOS, E. D. The effectof cultivar on critical periods of weed control in peanuts. Peanut Science, v.33, n.1, p.29-35, 2006.

CONAB.Companhia Nacional de Abastecimento. Grãos. Acompanhamento da Safra Brasileira de Grãos. Disponível em https://www.conab.gov.br/info agro/safras/graos> Acesso em: 20 abril, 2018.

EWRC. European Weed Research Council. Report of $3 \mathrm{rd}$ and 4rd meetings of EWRC. Committee of methods in weed research. Weed Research, v.4, n.1, p.88, 1964.

HEGDE, B. R.; VENUGOPAL, N.; FRIESEN, G. H. Chemical weed control in rainfed peanuts. Weed Research, v.17, n.5, p.325-328, 1977.

MAPA. Ministério da Agricultura, Pecuária e Abastecimento. Cultura com Suporte FitossanitárioInsuficiente. Registro de agrotóxicos para culturas com suporte fitossanitário insuficiente. Manual deprocedimentos (Instrução Normativa Conjunta 01, p. 24), 2015.

MARTINS, J.; DURIGAN, J. C.; APROBATO FILHO, A. Chemical weed control in groundnuts (Arachishypogaea). In: Resumos XIII Congresso Brasileiro de Herbicidas e Ervas Daninhas, Bahia, 1980. 1980.

NEPOMUCENO, M. P.; ALVES, P. L. C. A.; DIAS, T. C. S.; CARDOZO, N. P.; PAVANI, M. C. M. D. Efeito daépoca de semeadura nas relações de interferência entre uma comunidade infestante e a cultura do amendoim. Planta Daninha, v.25, n.3, p.481-488, 2007.

RAMAKRISHNA, A.; ONG, C. K.; REDDY, S. L. N. Efficacy of herbicides in the control of weeds in groundnut. International Arachis Newsletter, n. 7, p. 19-20, 1990. 
RODRIGUES, B. N.; ALMEIDA, F. S. Guia de herbicidas (6ª ed.). Londrina, PR, 2011, 697 p.

SANTOS, R. C.; FREIRE, R. M. M.; LIMA, L. M.; ZAGONEL, G. F.; COSTA, B. J. Produtividade de grãos e óleo de genótipos de amendoim para o mercado oleoquímico. Revista Ciência Agronômica, v.43, n.1, p.72-77, 2012.

ZANARDO, H. G.; CARREGA, W. C.; HIJANO, N.; CESARIN, A. E.; MARTINS, P. R. F. B.; GODOY, I. G.; ALVES, P. L. C. A. Herbicide Selectivity in Peanut Cultivars. Journal of Agricultural Science, v.10, n.8, p.447-456, 2018. 